cultured B cells improves their regulatory and or pro-inflammatory properties.

Materials and Methods Serum from SLE patients, RA patients (disease control) and normal controls was analysed for sema3A levels and was correlated with clinical parameters of SLE. The expression of sema3A on Bregs was compared between SLE patients and normal individuals. In addition to this, the expression of CD72 and TGF- $\beta$ (inhibitory molecules), and TLR-9 in B cells were assessed in both groups following the addition of recombinant sema3 $\mathrm{A}$ to cultured B cells.

Results 1. Serum sema3A levels were significantly lower in SLE patients comparing to that of RA patients and much lower than in normal controls $(55.04 \pm 16.30 \mathrm{ng} / \mathrm{ml}$ versus $65.54 \pm 14.82 \mathrm{ng} / \mathrm{ml}$, versus $74.41 \pm 17.60 \mathrm{ng} / \mathrm{ml}$, respectively, $\mathrm{P}<0.0001)$. 2. Sema3A levels were inversely correlated with SLE disease severity, kidney involvement and anti-cardiolipin-ab. 3. Sema3A expression on Bregs was significantly lower in SLE patients comparing normal individuals $(52.2 \pm 5.8 \%$ versus $82.6 \pm 6.4 \%, \mathrm{P}<0.0001$, respectively). 4 . The expression of both CD72 and TGF- $\beta$ was significantly decreased (37.88\%, 8.6\% respectively) in Bregs of SLE patients versus that on normal Bregs (49.26\%, 14.74\%, respectively; $\mathrm{P}=0.001)$. However, following the addition of sema3A to cultured B cells, a significant increase of TGF- $\beta$ and CD72 was noticed (altered in SLE patients, when compared to that of normal individuals). 5. The addition of sema3A to CpG-ODN stimulated B cells from SLE patients reduced TLR-9 expression by almost $50 \%$.

Conclusions 1 . This is the first study where sema3A is shown to be altered in both serum and on Breg cells of SLE patients. 2. Sema3A enhances the regulatory properties of Bregs, but this effect is shown to be altered in SLE. 3. Sema3A should be considered a future therapeutic tool in SLE.

\section{A5.29 SPONTANEOUS PRODUCTION OF ANTI-CITRULLINATED PROTEIN ANTIBODIES IN CULTURES OF PERIPHERAL BLOOD MONONUCLEAR CELLS AND SYNOVIAL FLUID MONONUCLEAR CELLS ISOLATED FROM PATIENTS WITH RHEUMATOID ARTHRITIS}

doi:10.1136/annrheumdis-2013-203219.29

Priscilla F Kerkman, Yoann Rombouts, Ellen IH van der Voort, Leendert A Trouw, Tom WJ Huizinga, René EM Toes, Hans U Scherer. Department of Rheumatology, Leiden University Medical Centre, Leiden, The Netherlands

Background and Objectives Anti-citrullinated protein antibodies (ACPA) are among the most important molecular candidates that could drive the inflammatory immune response in a subset of patients with rheumatoid arthritis (RA). So far, however, little is known on the phenotype and functional characteristics of ACPA producing B cells. Therefore, we studied ACPA producing B cells using ex-vivo cultures of peripheral blood mononuclear cells (PBMC) and synovial fluid mononuclear cells (SFMC).

Materials and Methods PBMC as well as SFMC from patients with ACPA-positive RA were cultured in 96 well plates without the addition of exogenous stimuli. Cultures were maintained for several weeks, with weekly complete replacement of the culture medium. Every week, supernatants were assessed for the presence of ACPAIgG and total IgG by ELISA. B cell subsets within the culture populations were determined by flow cytometry at several time points. Results Circulating, spontaneously ACPA producing B cells were readily detectable in peripheral blood of ACPA positive RA patients, but not in ACPA negative RA patients or healthy donors. FACS sorting experiments comparing isolated B cell subsets located spontaneous ACPA production to the plasmablast compartment. Memory $B$ cells were capable of ACPA production upon stimulation. In some culture wells, ACPA production was stable and detectable for up to 3 months. In a similar manner, we observed spontaneous, long-lasting ACPA production in (paired) SFMC cultures. The latter showed an up to 200 fold increase in ex vivo ACPA production compared to PBMC, but only a minor increase in the secretion of nonspecific IgG. B cell numbers in PBMC and SFMC were comparable in the starting population.

Conclusions ACPA specific plasmablasts circulate in the peripheral blood of patients with ACPA positive RA. Upon isolation, peripheral blood B cells can secrete ACPA spontaneously for several months. This observation suggests that ACPA specific B cells are either continuously recruited from the memory compartment, or that a subset of ACPA specific plasmacells might have the capacity to survive for extended periods of time. In SFMC, the frequency of ACPA specific $B$ cells is strongly increased. These observations point to a continuously active, ACPA specific immune response in RA.

\section{A5.30 SYSTEMIC INFLAMMATION AND B-CELLS IN RHEUMATOID ARTHRITIS}

doi:10.1136/annrheumdis-2013-203219.30

${ }^{1} \mathrm{M}$ Blits, 'S Vosslamber, 'J Lubbers, 'S de Ridder, 'AE Oostlander, ${ }^{3} \mathrm{GJ}$ Wolbink, ${ }^{3} \mathrm{D}$ van Schaardenburg, ${ }^{2,3} \mathrm{MT}$ Nurmohamed, 'DM Pegtel, 1,2CL Verweij. 'Department of Pathology, VU University Medical Center, Amsterdam, The Netherlands; ${ }^{2}$ Department of Rheumatology, VU University Medical Center, Amsterdam, The Netherlands; ${ }^{3}$ Department of Rheumatology, Jan van Breemen Research Institute | Reade, Amsterdam, The Netherlands

Background and Objectives Rheumatoid arthritis (RA) is heterogeneous in clinical symptoms, clinical parameters, pathogenesis and gene expression levels. Previously, we demonstrated variation in B-cell related gene expression between RA patients. The aim was to explore the relation of $\mathrm{B}$-cell related gene expression to clinical parameters of disease severity in early arthritis (EA) and established RA (esRA).

Methods B-cell related gene expression (B-cell score) was determined in peripheral blood cells of $26 \mathrm{EA}$ and 180 esRA patients, using multiplex real-time PCR. For the EA cohort, B-cell counts were also measured using flow cytometry. The esRA cohort was (randomly) divided into test and validation group of each $90 \mathrm{RA}$ patients, with a mean DAS28 of 5.0 and 5.2, respectively. Associations were assessed between $\mathrm{B}$-cell scores and the clinical disease parameters DAS28, CRP, RF, anti-CCP, nodules and erosions in all cohorts and B-cell counts only in EA cohort. Statistical testing was executed according to a bootstrap method which randomises the esRA group a 1000 times into two equally sized groups.

Results We demonstrated that the B-cell score reflected the peripheral blood B-cell count $(p<0.0001, r=0.7463)$. In EA, the B-cell score revealed a significant negative correlation with CRP levels $(p=0.0175$; $r=-0.4618)$. In the esRA group we also observed a negative correlation between the B-cell score and CRP levels $(p=0.0006, r=-0.3542$; $\mathrm{p}=0.0096$ after Benjamini-Hochberg multiple testing correction). This result was confirmed in the independent validation group $(p=0.0356 ; r=-0.2218)$. Additionally, we performed a randomisation with the bootstrap method, which showed the same significant correlations in almost all cases. However, no correlations were found between B-cell score and DAS28, RF, anti-CCP, nodules or erosions. Conclusions The B-cell score reflects the $\mathrm{B}$-cell count in RA and a low $\mathrm{B}$-cell count is associated with an increased marker of systemic inflammation in RA.

\section{A5.31 THE ROLE OF BOB1 IN RHEUMNATOID ARTHRITIS: POTENTIAL IMPLICATIONS FOR AUTOIMMUNITY}

doi:10.1136/annrheumdis-2013-203219.31

'Nataliya Yeremenko, 'Tineke Cantaert, 'Melissa van Tok, 'Ioana Gofita, 'Juan D Canete, 1,*Paul P Tak, ${ }^{3}$ Hergen Spits, 'Dominique Baeten. 'Academic Medical Center, University of Amsterdam, Amsterdam, The Netherlands; ${ }^{2}$ Hospital Clinic, Barcelona, Spain; ${ }^{3}$ Tytgat Institute for Liver and Intestinal Research, University of Amsterdam, Amsterdam, The Netherlands,

${ }^{*}$ Currently also: GlaxoSmithKline, Stevenage, UK 
Background Rheumatoid arthritis (RA) is a prototypic autoimmune disease characterised by a prominent humoral autoimmunity. Of particular relevance is the local production of autoantibodies such as rheumatoid factor and anti-citrullinated protein antibodies in the inflamed synovial tissue. The mechanisms underlying break of B cell tolerance and local autoantibody production remains poorly understood.

Objectives To identify cellular and molecular pathways implicated in RA-specific humoral autoimmunity.

Methods Synovial tissue samples were obtained by arthroscopy from untreated individuals with RA $(n=33)$ and inflammation matched SpA controls $(n=58)$. Gene expression profiling was performed on tissue samples of patients with established arthritis using 44K. Whole Genome Human microarrays (Agilent). Top differentially expressed genes were validated on three independent cohorts by Taqman based RT-qPCR and immunohistochemistry. Collagen-induced arthritis (CIA) and Experimental autoimmune encephalomyelitis (EAE) experiments were conducted using Bob1 knockout mice and their littermate controls.

Results Microarray screening for genes differentially expressed in the inflamed synovium, the key target of the disease process in RA, revealed a prominent and disease-specific $\mathrm{B}$ cell/plasma cell signature with the B cell-specific transcriptional co-activator Bob1 and its transcriptional target BCMA among the most upregulated genes. Validation by RT-qPCR on two independent cohorts representing early and established arthritis confirmed microarray data and demonstrated elevated expression of Bob1 and BCMA not only in established RA, but also at the early phase of the disease. Quantitative evaluation of immunohistochemical stainings of synovial tissue with monoclonal antibody for Bob1 revealed significant increase in Bob1 positive cells in RA synovium $(p<0.01)$. Next we determined whether lack of functional Bob1 modifies disease onset or severity in CIA. Interestingly, the results showed that Bob1 $1^{-/-}$mice were fully resistant to CIA induction compared to their wild-type littermates. This remarkable protection from CIA is explained by decreased antigen-presentation/costimulatory capacity of B cells and by failure to produce pathogenic anti-collagen autoantibodies in the absence of Bob1.

Conclusions The specific increase in Bob1 expressing cells in RA synovitis and the resistance of Bob1-deficient mice to development of CIA indicate that Bob1/BCMA axis may contribute to humoral autoimmunity in RA. The relationship between an aberrant Bob1 expression and the break of peripheral tolerance in RA is currently under investigation.

\section{Microbes and autoimmunity}

\section{A6.1 ANTIBODY RESPONSE AGAINST PORPHYROMONAS GINGIVALIS AND MATRIX METALLOPROTEINASE-3 ARE ASSOCIATED WITH ANTI-CITRULLINATED PROTEIN ANTIBODY IN RHEUMATOID ARTHRITIS, BUT ONLY MATRIX METALLOPROTEINASE-3 IS A PREDICTIVE FACTOR OF RESPONSE TO INFLIXIMAB}

doi:10.1136/annrheumdis-2013-203220.1

${ }^{1} \mathrm{M}$ Rinaudo-Gaujous, ${ }^{2} \mathrm{P}$ Miossec, ${ }^{3} \mathrm{~V}$ Blasco-Baque, ${ }^{4} \mathrm{P}$ Gaudin, ${ }^{5} \mathrm{~T}$ Thomas, ${ }^{1} \mathrm{~A}$ Moreau, ${ }^{1} \mathrm{C}$ Genin, ${ }^{1, *} \mathrm{~S}$ Paul, ${ }^{5, *} \mathrm{H}$ Marotte. 'Laboratory of Immunology and Immunomonitoring, CIC CIE3 Inserm Vaccinology, Hôpital Nord, CHU Saint-Etienne (France); ${ }^{2}$ Clinical Immunology Unit, Departments of Immunology and Rheumatology, Hôpital Edouard Herriot, CHU Lyon (France): ${ }^{3}$ Institute of Cardiovascular and Metabolic Diseases, CHU Rangueil (France); ' 4 Grenoble Teaching Hospital, CHU Hôpital Sud, Echirolles (France); ${ }^{5}$ Department of Rheumatology, Hôpital Nord, CHU Saint-Etienne (France),

*The authors contribute equally to this work

Background and Objectives This study evaluates clinical and biological markers of rheumatoid arthritis (RA) severity including matrix metalloproteinase (MMP-3) and Porphyromonas gingivalis (P. gingivalis) serology during infliximab treatment and highlights predictive factors of response to infliximab.
Materials and Methods Joint damage and severe periodontal disease were assessed in 101 RA patients included in this study. DAS28, anti-citrullinated protein antibodies (ACPA), anti-P. gingivalis antibody, and MMP-3 were monitored before and at 6 months of infliximab therapy. ACPA, anti-P. gingivalis antibody, and MMP-3 were determined by ELISA.

Results At baseline, ACPA titers were associated with anti- $P$. gingivalis LPS-specific antibodies titers $(\mathrm{P}<0.05)$. Anti- $P$. gingivalis antibodies were not significantly correlated with clinical, biological, or destruction parameters of RA disease. At 6 months of infliximab therapy, MMP-3 level decreased (from $119 \pm 103 \mathrm{ng} / \mathrm{ml}$ to $62.44 \pm$ $52 \mathrm{ng} / \mathrm{ml} ; \mathrm{P}<0.0001)$, whereas $P$. gingivalis antibody levels remained at the same level. DAS28 and inflammation markers (CRP and ESR) also decreased significantly during infliximab therapy $(\mathrm{P}<0.05)$ as ACPA levels $(\mathrm{P}<0.001)$. Only high MMP-3 level at baseline was associated with infliximab efficacy $(\mathrm{P}<0.01)$.

Conclusions MMP-3 level can be a useful marker of the efficacy of infliximab in RA patients. The treatment did not affect anti$P$. gingivalis antibodies.

\section{A6.2 INDUCIBLE TERTIARY LYMPHOID STRUCTURES AND AUTOIMMUNITY IN A NOVEL MODEL OF SIALOADENITIS IN WILD-TYPE MICE}

doi:10.1136/annrheumdis-2013-203220.2

'D Lucchesi, 'M Bombardieri, ${ }^{2} \mathrm{~F}$ Barone, ${ }^{2} \mathrm{~S}$ Nayar, ${ }^{3} \mathrm{G}$ Proctor, ${ }^{2} \mathrm{CD}$ Buckley, ${ }^{1} \mathrm{C}$ Pitzalis. ${ }^{1}$ William Harvey Research Institute, Experimental Medicine and Rheumatology, London, UK; 'University of Birmingham, Rheumatology Research Group, Birmingham, UK; ${ }^{3}$ King's College, Oral Medicine and Pathology, London, UK

Introduction Salivary glands of patients with Sjögren's syndrome (SS) develop ectopic lymphoid structures (ELS) characterised by $\mathrm{B} / \mathrm{T}$ cell compartmentalisation, the formation of high endothelial venules (HEV), follicular dendritic cell networks (FDCs), functional $B$ cell activation with expression of activation-induced cytidine deaminase (AID) as well as local differentiation of autoreactive plasma cells. The mechanisms triggering ELS formation, autoimmunity and exocrine dysfunction in SS are largely unknown. Here we present a novel model of inducible ectopic lymphoid tissue formation, breach of humoral self-tolerance and salivary hypofunction following delivery of a replication-deficient adenovirus-5 (AdV5) in submandibular glands of C57BL/ 6 mice through retrograde excretory duct cannulation.

Materials and Methods Luciferase- or LacZ-encoding Ad5 were delivered in C57BL/6 mice salivary glands (SG) through retrograde cannulation. SGs were collected at various time-points 1, 2 and 3 weeks post-cannulation ( $\mathrm{pc}$ ) and frozen sections were graded for infiltration and stained for T/B cell segregation, FDCs and HEV markers. Submandibular salivary flow was induced by pilocarpine stimulation and the amount of saliva measured. Expression of TLSrelated genes was investigated by TaqMan-PCR. Anti-viral antibodies and autoantibodies were detected by IF and western blot.

Results In this model, inflammation rapidly and consistently evolves from diffuse infiltration towards the development of SS-like periductal lymphoid aggregates within 2 weeks from AdV delivery. These infiltrates progressively acquire ELS features and support functional GL7+/AID + germinal centres. Formation of ELS is preceded by ectopic expression of lymphoid chemokines CXCL13, CCL2 1 and CCL19 as well as lymphotoxin- $\beta$ and is associated with development of anti-nuclear antibodies in $75 \%$ of the mice. Finally, reduction in salivary flow was observed over 3 weeks post AdV infection consistent with exocrine gland dysfunction as a consequence of the inflammatory response.

Conclusions This novel model has the potential to unravel the cellular and molecular mechanisms regulating ELS formation and their role in exocrine dysfunction and autoimmunity in SS. 